\title{
Multi-Parameter Based Scheduling for Multi-user MIMO Systems
}

\author{
K. Chanthirasekaran ${ }^{\dagger}$, M.A. Bhagyaveni* and L. Rama Parvathy**
}

\begin{abstract}
Multi-user multi-input multi-output (MU-MIMO) system has attracted the $4^{\text {th }}$ generation wireless network as one of core technique for performance enrichment. In this system rate control is a challenging problem and another problem is optimization. Proper scheduling can resolve these problems by deciding which set of user and at which rate the users send their data. This paper proposes a new multi-parameter based scheduling (MPS) for downlink multi-user multiple-input multiple-output (MU-MIMO) system under space-time block coding (STBC) transmissions. Goal of this MPS scheme is to offer improved link level performance in terms of a low average bit error rate (BER), high packet delivery ratio (PDR) with improved resource utilization and service fairness among the user. This scheme allows the set of users to send data based on their channel quality and their demand rates. Simulation compares the MPS performance with other scheduling scheme such as fair scheduling (FS), normalized priority scheduling (NPS) and threshold based fair scheduling (TFS). The results obtained prove that MPS has significant improvement in average BER performance with improved resource utilization and fairness as compared to the other scheduling scheme.
\end{abstract}

Keywords: Bit error rate (BER), Multi-Parameter based Scheduling (MPS), Multiple-input multipleoutput (MIMO), Packet delivery ratio (PDR)

\section{Introduction}

Multiuser multiple-input multiple-output (MU-MIMO) technology deployed to send message to multiple user without increasing transmit power or bandwidth in WLAN access point and in cellular base station. Downlink data rate of such system are linearly maximized with the number of transmitter antenna in rich multi path environment $[1,2]$. Proper scheduling in such system extracts multiuser diversity [3, 4]. Mostly, multi user scheduling scheme [5] used to schedule the user with the best channel quality. The scheduler of generalized selection combining (GSC) proposed in [6] select the Ks user with the largest SNR from the $\mathrm{K}$ active user (where $\mathrm{Ks} \leq \mathrm{K}$ ) in each time-slot. A scheduler proposed in [7] interacts with the physical layer for sum rate improvement. Proportional Fair Scheduling (PFS) algorithm shown in [8] provides fairness among users instead of simply selecting best users. Open loop transmit diversity of a simple proportional fair scheduler exhibit poor performance as compared to closed loop transmit diversity scheme under wide range of mobility condition studied by Berger et al [9]. Multi-user diversity gain extracted by opportunistic scheduling proposed for

$\dagger$ Corresponding Author: Dept. of Electronics and Communication Engineering, Anand Institute of Higher Technology, Chennai, India. (ece kc@yahoo.com)

* Dept. of Electronics and Communication Engineering, College of Engineering, Anna University, Guindy, Chennai, Tamilnadu, India. (bhagya@annauniv.edu)

* Dept. of Computer Applications, SSN College of Engineering, Chennai, India. (ramaparvathyl@ssn.edu.in)

Received: September 27, 2013; Accepted: July 21, 2015 single-antenna multi-user system [6] and then extended for multiple-antenna system [10]. A simple multiuser switched scheme is proposed in [14] to simplify the user selection process. Work on linking spatial diversity with multiuser diversity is proposed in [15]. In this scheme the user whose channel condition is higher than the predetermined threshold value is scheduled. In the existing MAC layer downlink scheduling, despite MIMO advantages, we see the performance gap among the max-min fair scheduling presented in [11], proportional fair scheduling and threshold based fair scheduling shown in [12] and [16]. Finding the threshold is crucial for system performance and resource utilization. These issues were not well addressed in [16]. This paper proposes a new multiparameter based scheduling to fill the gap between the existing scheduling. This scheduler allocates resource based on user channel quality and their demand data rate. First the scheduler grants resource for the set of user whose channel quality is high and their demand rate is low, then it grants resource for the another set of user whose channel quality is high and their demand rate is medium. This way this scheduler grants resource to set user according to case Table 1 . Then, we compare the proposed scheduler performance with other scheduler like fairness scheduler (FS), normalized SNR scheduler and threshold based scheduler (TFS).

This paper organized as follows. Section II presents system overview. Section III describes various scheduling algorithms. Section IV discusses the simulation results of the new resource allocation scheme and Section V offers concluding remarks. 


\section{System Model}

In this section, downlink multiuser MIMO system [17] with different scheduler (MPS) is modeled with single base station (BS) shown in Fig. 1. Let 'L' be the pair of transmit antenna available at base station. And ' $N$ ' is the number of active mobile user each with pair of receive antenna. Here the BS scheduler probes all ' $N$ ' user channel state information. Base station scheduler schedule the active user based on their channel quality and demand rate. Then it grants available resources to the scheduled user. The BS uses 2X2 Alamouti -STBC code in physical layer.

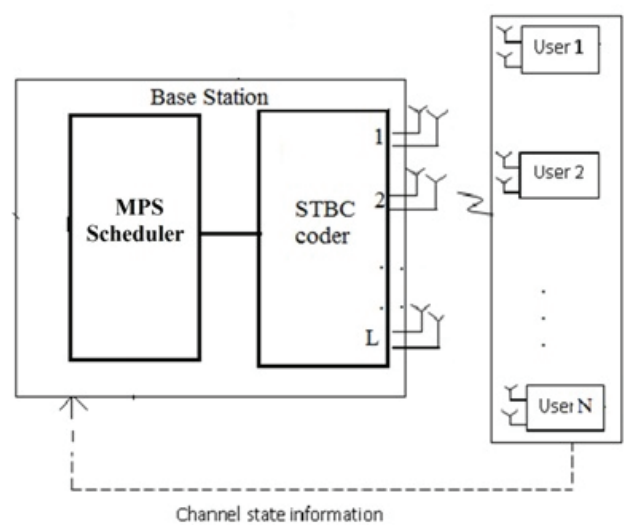

Fig. 1: Downlink model of MU-MIMO system

It is assumed that the channel quality stays stationary during a time slot. User scheduling and resource granting process is carried during the guard period. In subsequent data burst the resource granted user performs data transmission.

\section{Scheduling Algorithms}

We assume that the base station knows the channel state information of all active users. It can select a group of user from the entire active user to achieve the better performance with the help of various scheduling. Here the equal number of transmit antenna (two) are allotted per user in the time slot among active user by considering the number of resource available. The following scheduling algorithms are used in the scheduler at the base station for performance comparison.

\subsection{Fair Scheduling (FS)}

Let ' $\mathrm{N}$ ' be the total number of active users who send their channel state information (CSI) to BS in a time slot $t_{k}$ Let ' $L$ ' be the total number of resources available at the base station. This scheduler grants resources based on first come first serve (FCFS) basis. Let $\left\{x_{1}, x_{2}, x_{3}, \ldots x_{L} . . x_{N}\right\}$ be the active users in the BS at time slot $t_{k}$ as fair scheduler grant resource based on first come first serve basis, first $\mathrm{L}$ number of users is scheduled by this scheduler. This scheduler maintains the service fairness but the average BER performance of the system under this scheduling is poor. In order to improve the average BER performance normalized priority scheduling is modeled in [13].

\subsection{Normalized priority scheduling}

Normalized priority scheduler receives SNR information from all users at each time slot $t_{k}$ and it computes normalized SNR of each user which is based on this user's previous slot SNR value. User's SNR value is normalized as and when user's SNR value is changed. Then it assigns resource based on these normalized SNR value. Hence improved level of fairness is incorporated as compared to priority scheduling (PS). The granted users transmit their data by Alamouti -STBC coded 2X2 MIMO system. This normalized priority scheduling achieves BER performance similar to priority scheduling (PS) along with improved service fairness. To achieve better fairness among users threshold based fair scheduling has described.

\subsection{Threshold based Fair Scheduling (TFS)}

Threshold based fair scheduler proposed in [12] is based on "on-off based scheduling(OOBS)" proposed in [16] that computes offer-able SNR threshold 'Th' by using average of minimum SNR and maximum SNR of active user. Then ' $\mathrm{R}$ ' resources are granted to the users in the time slot $t_{k}$ based on first come first serve if their SNR $\geq$ 'Th'. Let $x=\left\{x_{1}, x_{2}, x_{3}, \ldots . x_{N}\right\}$ be the users request received at time slot $t_{k}$ and Let $x^{\prime}=\left\{x_{1}^{\prime}, x_{2}^{\prime}, x_{3}^{\prime} \ldots \ldots . x_{p}^{\prime}\right\}$ be the users whose SNR's are greater than or equal to 'Th' where $x^{\prime} \subset x$. Let ' $p$ ' be the total number of scheduled user. The granted users $G_{t_{k}}$ in the time slot $t_{k}$ is given as

$$
G_{t_{k}}= \begin{cases}\left\{x_{1}^{\prime}, x_{2}^{\prime}, x_{3}^{\prime} \ldots \ldots . x_{L}^{\prime}\right\} & \text { if } p \geq L \\ \left\{x_{1}^{\prime}, x_{2}^{\prime}, x_{3}^{\prime} \ldots \ldots . x_{p}^{\prime}\right\} & \text { if } p<L\end{cases}
$$

This TFS scheme provides better link level performance but at the cost of poor resource utilization. In this scheduling the user is scheduled if their channel strength exceeds required threshold value. In MU-MIMO system, if number of user scheduled by this scheduler is less compared to $\mathrm{L}$ resource, then the remaining resource become unutilized during the particular time slot. A new multi-parameter based user scheduling (MPS) algorithm is developed for improving the system performance and service fairness.

\subsection{Multi-Parameter based Scheduling (MPS)}

Basically, TFS considers only one user parameter (i.e. channel quality) for scheduling. Also it considers one group of user (i.e. the user's SNR is above threshold) whereas Multi-parameter based user scheduler schedules 
the user based on users' SNR and their demand rate. During guard period this scheduler receives the information about each user channel quality and their demand data rate. Using this user's values the scheduler computes three equal SNR regions $\mathrm{SNR}_{\mathrm{L}}, \mathrm{SNR}_{\mathrm{M}}$ and $\mathrm{SNR}_{\mathrm{H}}$ in every time slot that is based on maximum SNR value of the current active users as given in Eq. 2. Similarly it computes three equal rate regions Rate $_{\mathrm{L}}$, Rate $_{\mathrm{M}}$, and Rate $\mathrm{H}_{\mathrm{H}}$ in every time slot as given in Eq. 3. In this simulation minimum user SNR to be served is assumed to be $1 \mathrm{db}$. If any user SNR is less than $1 \mathrm{db}$, it will not be taken into consideration for scheduling since those user channel quality will be very poor (more noise or interference or fading than signal).

$$
\begin{aligned}
& \mathrm{SNR}_{\mathrm{L}}<\max (\mathrm{SNR}) / 3, \\
& \max (\mathrm{SNR}) / 3 \leq \mathrm{SNR}_{\mathrm{M}}<2 \times \max (\mathrm{SNR}) / 3, \\
& 2 \times \max (\mathrm{SNR}) / 3 \leq \mathrm{SNR}_{\mathrm{H}} \leq \max (\mathrm{SNR}) \\
& \operatorname{Rate}_{\mathrm{L}}<\max (\text { Rate }) / 3, \\
& \max (\text { Rate }) / 3_{\mathrm{L}} \leq \text { Rate }_{\mathrm{M}}<\max (\text { Rate }) / 3, \\
& 2 \times \max (\text { Rate }) / 3 \leq \text { Rate }_{\mathrm{H}} \leq \max (\text { Rate })
\end{aligned}
$$

Then it schedules the user based on case described in the Table 1. Fig. 2 shows the MPS scheduling flow diagram.

Table 1. MPS Scheduler case table

\begin{tabular}{c|c|c|c|c|c|c}
\hline Case $\mathrm{i}$ & 1 & 2 & 3 & 4 & 5 & 6 \\
\hline SNR & $\mathrm{H}$ & $\mathrm{H}$ & $\mathrm{M}$ & $\mathrm{M}$ & $\mathrm{H}$ & $\mathrm{M}$ \\
\hline Rate & $\mathrm{L}$ & $\mathrm{M}$ & $\mathrm{L}$ & $\mathrm{M}$ & $\mathrm{H}$ & $\mathrm{H}$ \\
\hline
\end{tabular}

L - Low, M- Medium, H - High

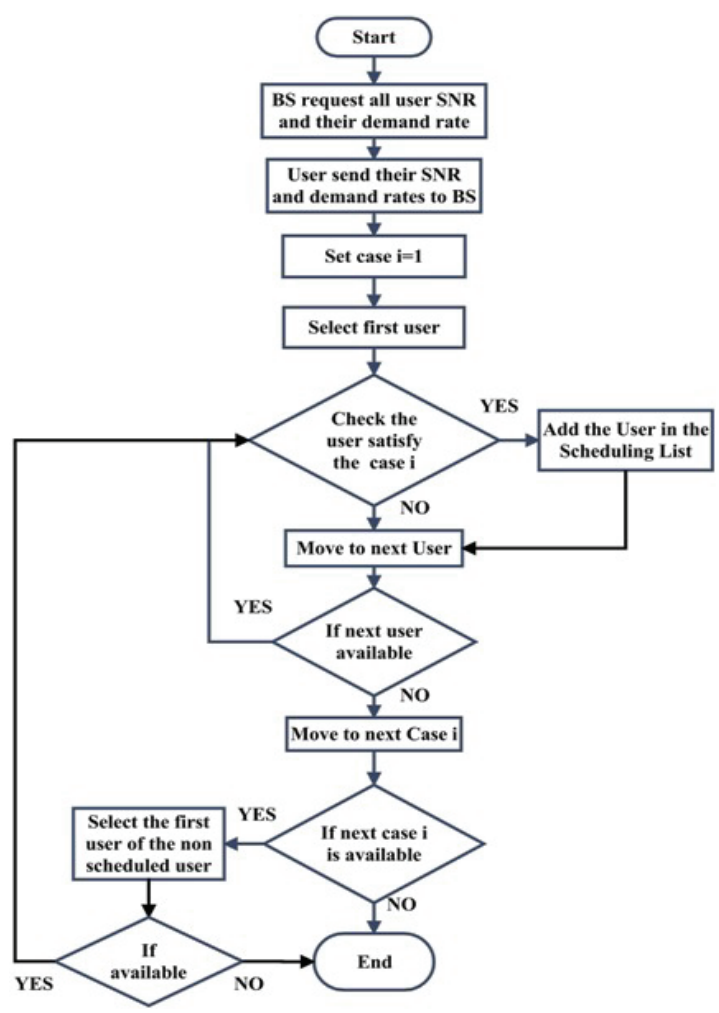

Fig. 2: Flow diagram of MPS
Fig. 2 clearly describes that the base station probes all the users channel strength along with their demand data rate. Then the base station scheduler computes different SNR region and Rate regions. During scheduling process, MPS scheduler selects first case given in Table 1. It selects the first active user and verifies the user comes under the particular case. If yes, the scheduler moves the particular user to the scheduled user list and select next unscheduled user and verify the same. If no, the scheduler selects the next unscheduled user and verifies the same.

Then the scheduler selects the next case and does the same scheduling process till last case. Therefore the MPS first schedules the users who come under $\mathrm{SNR}_{\mathrm{H}}$, Rate region, and then it schedules the users who come under $\mathrm{SNR}_{\mathrm{H}}$, Rate $\mathrm{M}$ region. Similarly MPS schedules user according to the case described in case Table 1. It is clear from case Table 1 that MPS makes the most of the minimum demand data rate user traffic flow whose channel quality is good at first. Consequently, it schedules the other user. The MPS scheduling algorithm is given below.

Let $x=\left\{x_{1}, x_{2}, x_{3}, \ldots . x_{N}\right\}$ be the users request received at time slot $t_{k}$, let $x^{\prime}=\left\{x_{1}^{\prime}, x_{2}^{\prime}, x_{3}^{\prime} \ldots \ldots . x_{q}^{\prime}\right\}$ be the MPS scheduled users where $x^{\prime} \subset x$ and let ' $\mathrm{q}$ ' be the total number of MPS scheduled user. The granted users $G_{t_{k}}$ in the time slot $t_{k}$ is given as

$$
G_{t_{k}}= \begin{cases}\left\{x_{1}^{\prime}, x_{2}^{\prime}, x_{3}^{\prime} \ldots \ldots . x_{L}^{\prime}\right\} & \text { if } q \geq L \\ \left\{x_{1}^{\prime}, x_{2}^{\prime}, x_{3}^{\prime} \ldots \ldots . x_{p}^{\prime}\right\} & \text { if } q<L\end{cases}
$$

\footnotetext{
Begin; - Initiate scheduler in Base Station

Probe SNR $(\gamma)$ information for all users $(\mathrm{N})$ by CSI

Probe rate request $(r)$ for all users

Construct case table by using $\operatorname{rate}(r), \operatorname{SNR}(\gamma)$

1. Define L,M,H Values $\forall \gamma$,

$$
\begin{aligned}
& \text { Split regions } \gamma=1: L: M: H \text {, where } \gamma>0 \\
& L=1:\left(\gamma_{\text {high }} / 3\right) ; M=\left(\gamma_{\text {high }} / 3\right): 2\left(\gamma_{\text {high }} / 3\right) ; H=2\left(\gamma_{\text {high }} / 3\right): \gamma_{\text {high }}
\end{aligned}
$$
end;

2. Define $L, M$, $H$ Values $\forall r$,

Split regions $r=1: L: M: H$, where $r>0$

$$
r=1:\left(r_{\text {high }} / 3\right) ; M=\left(r_{\text {high }} / 3\right): 2\left(r_{\text {high }} / 3\right) ; H=2\left(r_{\text {high }} / 3\right): r_{\text {high }}
$$$$
\text { end; }
$$

3. Create regions with pair $R(\gamma, r)$

4. Rank the regions,

For better performance and fair usage, SNR to be high and Rate is low

Rank in $\operatorname{order} R(\gamma, r)=\{H L ; H M ; M L ; M M ; H H ; M H\}$

Create scheduling list concurrence with region pairs for Region $j=1: R$

$$
\begin{aligned}
& \text { for user } i=1: N \\
& \quad \text { if } r_{i}, \gamma_{i} \in R_{j} \\
& \% \text { Add user to the scheduled user list } \% \\
& \text { user } i \subset \text { scheduled user list } \\
& \text { end; }
\end{aligned}
$$

end;

Allocate resources to users as per scheduled user list 


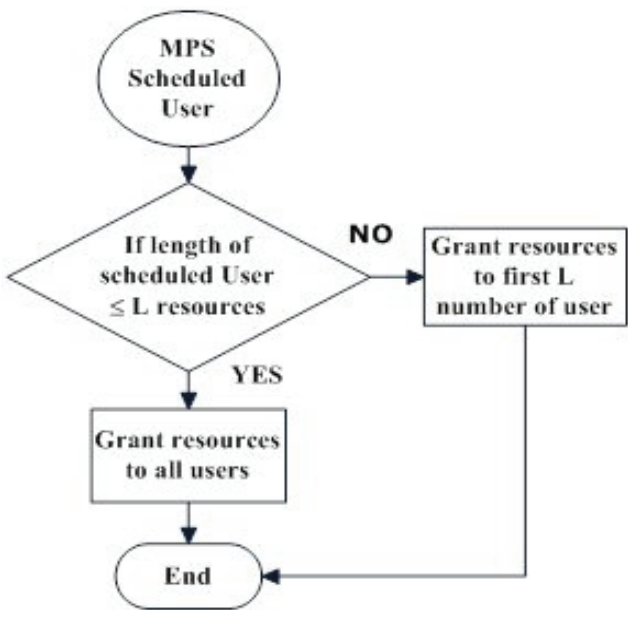

Fig. 3: MPS Resource allocation

MPS resource granting flow diagram is shown in Fig. 3. This is a modified MTCAS resource allocation flow diagram [17]. Here MPS scheduled user is according to MPS scheduling.

This scheduler considers the user who comes under six regions for performance enrichment and resource utilization instead of one SNR threshold considered in TFS. The idea of this scheduling is to combine max-min scheduling; priority scheduling and threshold-based scheduling. If none of the users are in scheduled list, the scheduler waits for next guard time. If all users are in the high SNR region and low Rate region, the scheduler achieves full fairness and acts as fair scheduler during that time. Since this scheduler maximizes the minimum date rate user flow whose channel quality is good, it achieves the BER improvement and it achieves better resource utilization because it considers many user regions.

The performance of the proposed scheduler is compared with other scheduling policies like fair scheduling (FS), priority scheduling (PS), and threshold-based fair scheduling (TFS) using simulation.

\section{Performance Measure}

In the simulation the following measure has been carried out.

\subsection{Bit Error Rate (BER) measurement}

BER of a wireless communication system is defined as the ratio of number of error bits and total number of bits transmitted during a specific period. It is the likelihood that a single error bit will occur within received bits, independent of rate of transmission.

\subsection{Packet Delivery Ratio (PDR)}

PDR of a wireless network is defined as the ratio of received packets with respect to transmitted packets. In this work, we have considered 64 bytes as one packet and number of bits taken for simulation is $10^{6}$.

\subsection{Fairness}

As far as fairness measure is concerned, we take the order of service into consideration. If the users are served on first come first serve (FCFS) basis, then it is a full fair scheduler.

\subsection{Resource utilization}

Resource utilization is a measure of the percentage of available antenna resource which has been allocated for transmission. It is defined as the ratio of number of resource granted by the scheduler for active user with respect to total number of available resource at the base station.

\section{Simulation Results}

The system is modeled using one base station, ' $\mathrm{N}$ ' number of active users and ' $L$ ' number of resource available at base station. The BS receives all active users' SNR and their demand rate. The performance of scheduling is simulated under each user has 2 antennas and demand 2 antenna resources from the base station. In this $2 \times 2 \mathrm{MU}-$ MIMO system the user data's are transmitted via Rayleigh channel after Alamouti - Space Time Block Coding (STBC). Simulation parameters [17] are shown in Table 2.

Table 2. Simulation parameters for MIMO-STBC system

\begin{tabular}{c|c}
\hline System & MIMO-STBC \\
\hline Number of Transmit antenna & 2 \\
\hline Number of Receive antenna & 2 \\
\hline Channel & Rayleigh flat fading \\
\hline Noise & AWGN \\
\hline Modulation & BPSK \\
\hline Number of active user $\mathrm{N}$ & 25 \\
\hline
\end{tabular}

For this simulation user SNR is chosen between $1 \mathrm{db}$ and $25 \mathrm{db}$. Because any user whose channel is considered to be noisier if their channel strength is lower than $1 \mathrm{db}$ and maximum boundary is chosen as saturation point beyond which the link level performance is almost flat. Since this MPS scheduler maximizes the minimum demand rate user whose channel quality is good, the scheduler achieves improved BER performance. Also this scheduler consider six user regions described in Table 1, there will be improved level of resource utilization as compared to threshold based fair scheduler.

The BER performance of MPS is shown in Figs. 4 and 5 under user variation with number of available resource $\mathrm{L}$ $=6$ and 9 respectively It shows clearly that when the 


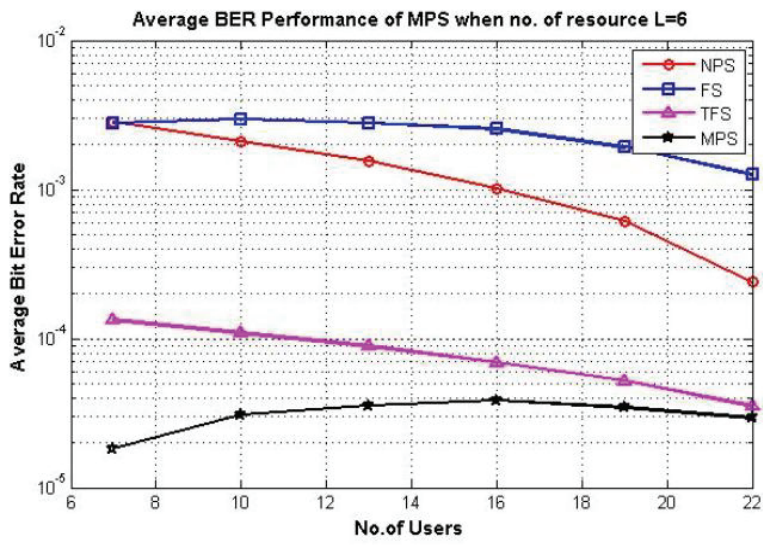

Fig. 4. BER performance comparison of MPS with $L=6$ with other scheduling

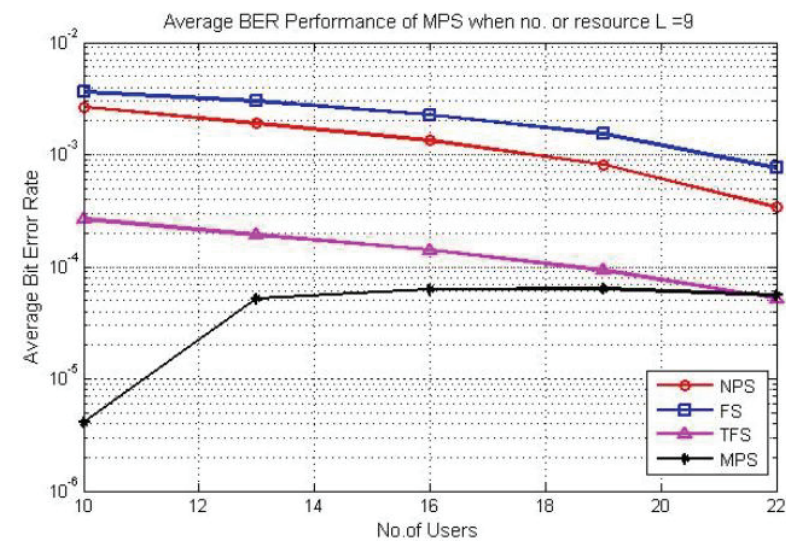

Fig. 5. BER performance comparison of MPS with $L=9$ with other scheduling

number of user increases, there is a chance of selecting user till the $\left(\mathrm{SNR}_{\mathrm{M}}, \mathrm{Rate}_{\mathrm{H}}\right)$ region and hence resources are utilized properly at the cost of small degradation in BER performance. When there is less number of active users, i.e. closer to $\mathrm{L}$, there will be more chance to schedule the number of best user according to case table and, hence, BER performance is improved. From the Figs. 4 and 5, it is clear that MPS out performs threshold based fair scheduler. The BER performance of MPS becomes closer to TFS when the number of users is more compared to available resources. From Fig. 4, it is observed that the average BER performance of MPS is $3 \times 10^{-5}$ when $\mathrm{L}=6$ and $\mathrm{N}=22$. This is around $25 \%$ improvement compared to TFS.

The Simulation results of BER values of MPS with $\mathrm{L}=6$ are shown in Table 3. From Table 3, it is observed that MPS always outperforms TFS and NPS. It is observed that MPS gives best BER performance when the active users are closer to available resource ( $\mathrm{L}=80 \%$ of users) and there will be a small degradation in BER performance in other cases.

When the number of resource available is closer to number of active users $(\mathrm{L}=80 \%$ of active user. i.e. $\mathrm{L}=6$ and $\mathrm{N}=7$ ), there is a chance of scheduling less number of best users come under MPS case table. Hence MPS
Table 3. Simulation results of BER values of MPS with different schedulers

\begin{tabular}{c|c|c|c|c}
\hline Scheduler & FS & NPS & TFS & MPS \\
\hline Resource & $2 \times 10^{-3}$ & $5 \times 10^{-4}$ & $5 \times 10^{-5}$ & $3 \times 10^{-5}$ \\
\hline $\mathrm{L}=30 \%$ & $3 \times 10^{-3}$ & $2 \times 10^{-3}$ & $10^{-4}$ & $3 \times 10^{-5}$ \\
\hline $\mathrm{L}=60 \%$ & $2 \times 10^{-3}$ & $2 \times 10^{-3}$ & $2 \times 10^{-4}$ & $2 \times 10^{-5}$ \\
\hline $\mathrm{L}=80 \%$ &
\end{tabular}

performance is best under this region by sacrificing the little resource utilization. Under the resource constrined suituation (i.e $\mathrm{L}=60 \%$ or $30 \%$ of number of users), there is chance of scheduling more number of users, hence resouce utilization increases and BER performance of MPS is little reduced.

The BER performance of MPS under resource variation is shown in Fig. 6. It shows that the multi-parameter based scheduling performs well compared to normalized priority scheduling when the number of resources exceeds 59 percent of number of users. Otherwise, NPS gives better performance because it serves the group of best users. When the available resource increases and becomes closer to the number of users, normalized priority scheduling gives the same performance as FS. Also it is clear that the MPS outperforms TFS due to MPS first schedule $\mathrm{SNR}_{\mathrm{H}}$ region users who transmit lesser amount of data.

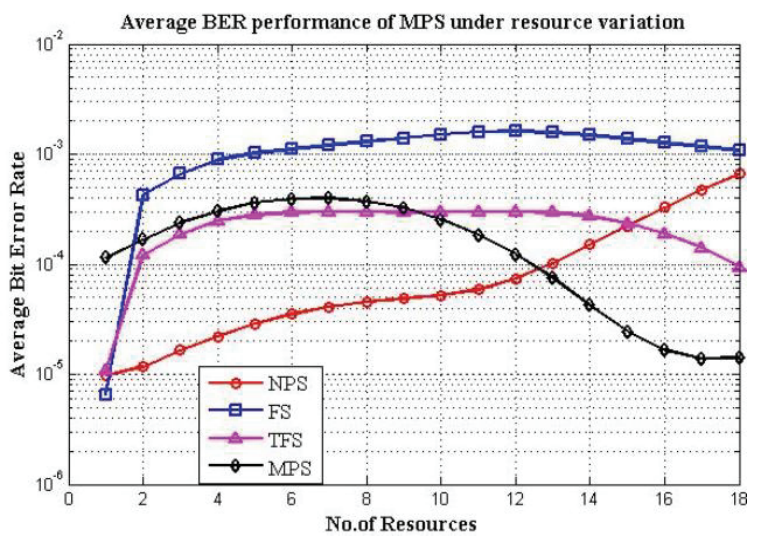

Fig. 6. BER performance comparison of MPS with other scheduling under resource variation

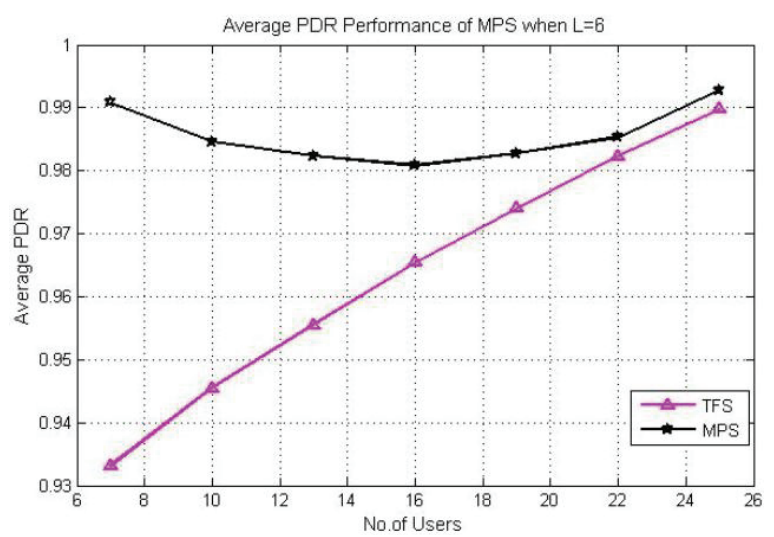

Fig. 7. PDR performance comparison of MPS with $L=6$ with TFS 
The packet delivery ratio (PDR) of this scheme under active user variaton with $L=6 \& 9$ is shown in Figs. 7 and 8 respectively. Since this scheduler maximizes the minimum data rate flow, the packet delivery ratio of this scheme will be better than TFS. This is shown in Figs. 7 and 8.

The packet delivery ratio (PDR) of this scheme under resource varion is shown in Fig. 9. The PDR performance of MPS scheme is found improved compared to the TFS

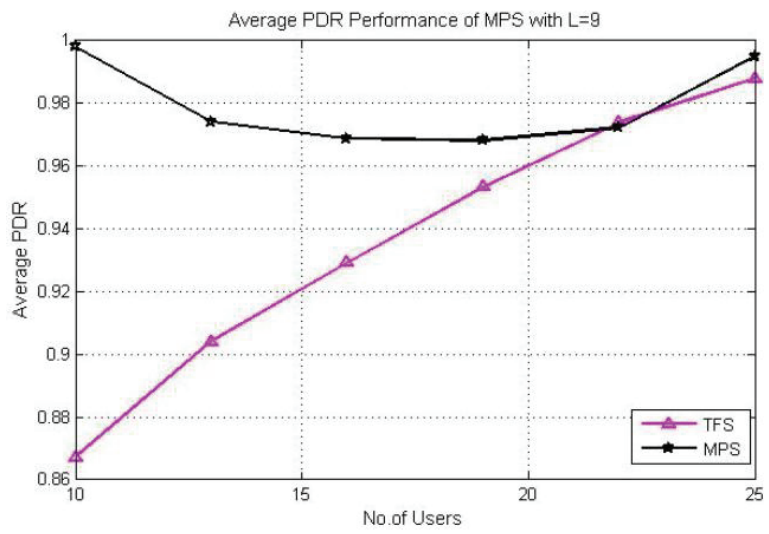

Fig. 8. PDR performance comparison of MPS with $L=9$ with TFS

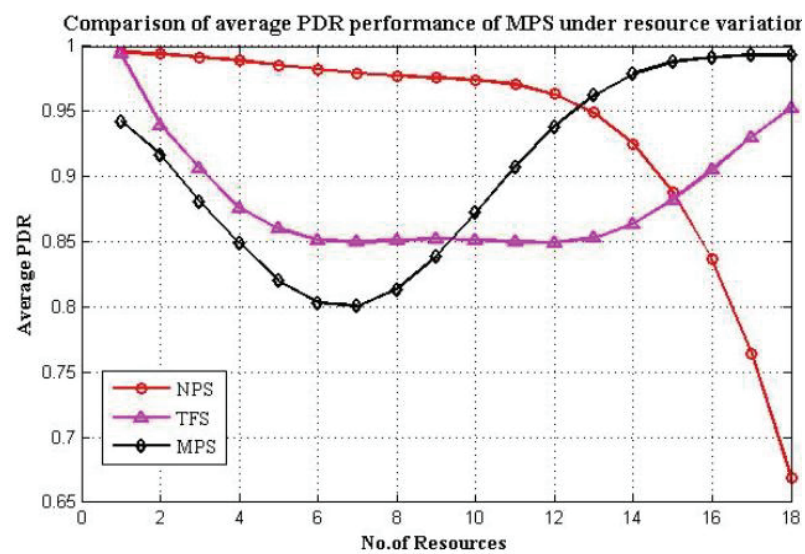

Fig. 9. PDR performance comparison of MPS with NPS \& TFS under resource variation

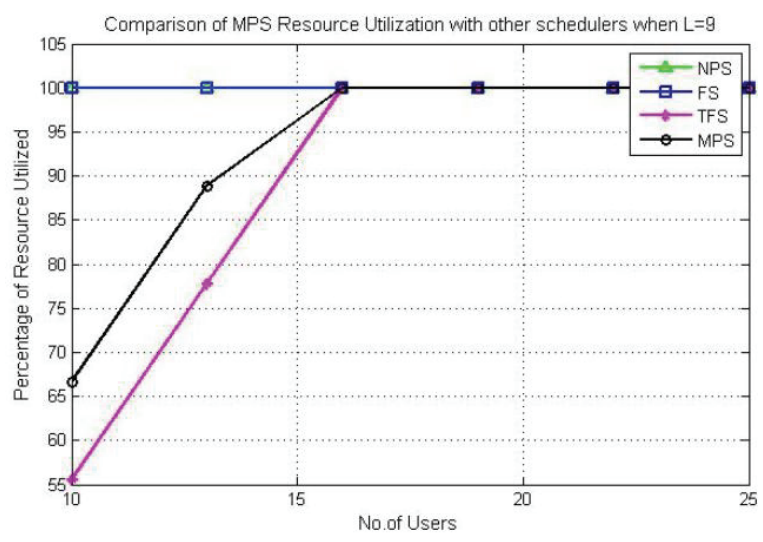

Fig. 10. Comparison of MPS resource utilization performance when $\mathrm{L}=9$ scheme and less as compared to NPS when number of resources is less than 59 percent of the number of users. Also it is observed that when the number of resources is greater than 59 percent of the number of users, the PDR performance of NPS deeply decreases and the MPS is able to maintain above $95 \%$ of PDR performance.

Resource utilization of MPS is shown in Fig. 10 and it is found that MPS resource utilization is improved compared to TFS.

When the number of available resource is less than or equal to 56 percent of active users, all schedulers utilize the entire available resource. In other regions, MPS resource utilization is improved as compared to TFS. When $\mathrm{L}=9$ along with $\mathrm{N}=13$, MPS resource utilization is $89 \%$ and TFS resource utilization is $77 \%$. From Fig. 9, it is also observed that MPS resource utilization performance is improved by $12 \%$ compared to TFS at this point.

\section{Conclusion}

This paper has described multi-parameter based scheduler that works based on active users' channel strength and their demand data rate. This scheduler combines the max-min scheduler, priority scheduler and threshold based scheduler. The performances of this scheme with BPSK modulations in flat Rayleigh fading channels is compared with other scheduling schemes such as FS, NPS and TFS. From the simulation results it is found that MPS outperforms other scheduling schemes in average BER, PDR performance. This scheme provides a network BER of about $3 * 10^{-5}$ and PDR of $97 \%$. Also it is observed that the average BER performance of MPS is $3 * 10^{-5}$ when $\mathrm{L}=6$ and $\mathrm{N}=22$. This is around $25 \%$ improvement compared to TFS. MPS resource utilization is $89 \%$ when $\mathrm{L}=9$ and $\mathrm{N}=13$. This is improved by $12 \%$ compared to TFS at this point. This work can be extended by implementing this MPS scheduler in cooperated multiple base station MIMO network.

\section{Acknowledgements}

This work was supported by the DRDO Research Lab, Department of ECE, Anand Institute of Higher Technology Chennai, India.

\section{References}

[1] S. Vishnawath, N. Jindal, and A. Goldsmith, "Duality, achievable rates and sum capacity of Gaussian MIMO channels," IEEE Trans. Inf. Theory, vol. 49, no. 10, pp. 2658-2668, Oct. 2003.

[2] N. Jindal and A. Goldsmith, "Dirty-paper coding versus TDMA for MIMO broadcast channels," IEEE Trans. Inf. Theory, vol. 51, no. 5, pp. 1783-1794, May 
2005.

[3] Jalali, R. Padovani, and R. Pankaj, "Data throughput of CDMAHDR, a high efficiency high data rate personal communication wireless system," Proc. IEEE Veh. Technol. Conf., pp. 1854-1854, May 2000.

[4] X. Liu, E.K.P. Chong, and N.B. Shroff, "Opportunistic transmission scheduling with resource-sharing constraints in wireless networks," IEEE J. Sel. Areas Commun., vol. 19, no. 10, pp. 2053-2064, Oct. 2001.

[5] D. N. Tse, "Multiuser diversity in wireless networks," Apr. 2001, presentation, Stanford University.

[6] Y. Ma and D. Zhang, "Performance of generalized selection multiuser scheduling over generalized fading channels," in Proc. IEEE Int. Wireless Commun. and Mobile Comput. Conf. (IWCMC'06), Vancouver, Canada, July 2006.

[7] J. Jiang, R. M. Buehrer; W. H Tranter; "Antenna diversity in multiuser data Networks", IEEE Transactions on Communications, Volume 52, Issue 3, March 2004, Page(s):490-497.

[8] P. Visawanath, D. N. C. Tse, and R. Laroia, "Opportunistic Beamforming Using Dumb Antennas," IEEE Trans. Info. Theory, vol. 48, no. 6, June 2002, pp. 1277-94.

[9] L.T. Berger, T.E. Kolding, J.R.Moreno, P.Ameigeiras, L. Schumacher, P. E. Mogensen, "Interaction of Transmit Diversity and Proportional Fair Scheduling", IEEE Vehicular Technology Conference, 2003, Vol 4, Page(s):2423 - 2427.

[10] M. K. Simon and M.-S. Alouini, "Performance analysis of generalized selection combining with threshold test per branch (T-GSC)," IEEE Trans. Veh. Technol., vol. 51, no. 5, pp. 1018-1029, 2002.

[11] D. Bertsekas and R. Gallagar, Data Networks. Englwood Cliffs, N.J.: Prentice-Hall, 1992.

[12] K.Chanthirasekaran, Dr. M.A. Bhagyaveni, “An Efficient Scheduling Scheme for Resource Utilization and Performance Enhancement of Multi User MIMO Wireless Systems" Information Technology Journal, Vol. 10, no.7, 2011, pp 1452-1456.

[13] L. Yang and M.-S. Alouini, "Performance analysis of multiuser selection diversity," in IEEE Transactions on Vehicular Technology, Volume: 5 , Issue: 6, (2006), pp: $1848-1861$

[14] Holter, M.-S. Alouini, G. E. Øien, and H.-C. Yang, "Multiuser switched diversity transmission," in Proc. IEEE Veh. Technol. Conf., vol. 3, Los Angeles, CA, USA, (Sep. 2004), pp. 2038-2043

[15] H. Nam, Y.-C. Ko, and M.-S. Alouini, "Performance analysis of joint switched diversity and adaptive modulation," IEEE Trans. Wireless Communication., vol. 7, no. 10, (Oct. 2008), pp. 3780-3790

[16] Sung Sik Nam, Mohamed-Slim Alouini, Hong-Chuan Yang and Khalid A. Qaraqe, "Threshold-Based Parallel Multiuser Scheduling" in IEEE Transactions On Wireless Communications, vol. 8, no. 4, (April 2009), pp. 2150-2159.

[17] K.Chanthirasekaran, Dr. M.A. Bhagyaveni, "MultiThreshold based Scheduling for Multi-User MultiInput Multi-Output Systems" Journal of Computer Science, Vol. 8, no. 7, 2012, pp. 1171-1176.

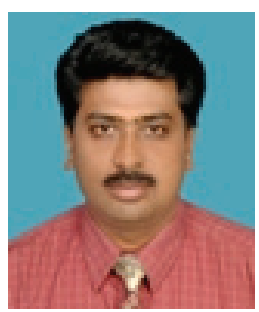

K. Chanthirasekaran $\mathrm{He}$ received $\mathrm{PhD}$. degree in Information and Communication engineering from Anna University. His research interests are MIMO wireless networks, Scheduling and Mobile Adhoc Networks.

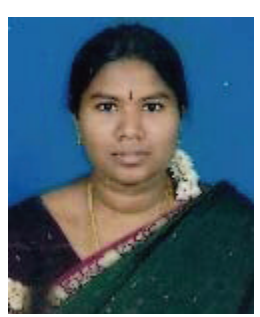

M. A. Bhagyaveni She is working as Professor in the Department of Electronics and Communication Engineering, Anna University. Her field of interest includes Wireless networks and Sensor Networks.

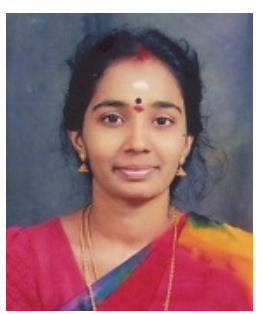

L. Rama Parvathy She is working as Associate Professor in the Department of Computer Application, SSN College of Engineering, Engineering. She has obtained her Ph.D. in the field of Grid computing. Her field of interest includes Grid Computing and Scheduling. 